\title{
Diagnostics of the technical condition of transformers according to the color characteristics of transformer oil
}

\author{
$D M$ Valiullina, $Y u$ K Ilyasova* and $V K$ Kozlov \\ Department of electric power systems and networks, Kazan State Power Engineering University-KSPEU, Kazan, Russian Federation
}

\begin{abstract}
This paper presents the results of a research of transformer oil in a visual way. The high information content of the optical radiation scattered and transmitted through the oil is shown. By the color of the radiation, it is possible to determine such characteristics of the oil as acid number, dielectric loss tangent, the presence of colloidal particles, their concentration and size, as well as the presence of aromatic compounds in the oil, changes in their molecular composition and concentration. By the presence of aromatic compounds and colloidal particles, it is possible to visually state the development of thermal and discharge defects in a transformer. This diagnostic system allows to assess the condition of power transformers without shutting them down, make decisions on their further operation without the use of expensive equipment and at the initial stage to identify the changes that are significant and necessary. This is due to the high cost of the transformer, its importance in terms of the reliability of power supply to consumers, the complexity of determining damages and defects at an early stage of development.
\end{abstract}

\section{Introduction}

Nowadays special attention is paid to the reliability of the electrical equipment of power systems, especially power transformers. The power transformer in the power system is one of the most important elements that determine the reliability of power supply. The sudden failure of a transformer causes great damage, because losses are associated not only with the need to restore the transformer, but also with a break in the supply of electricity [1-9]. Reliable operation of a power transformer is determined by the technical condition of all its components and elements, including the insulating oil. One of the main ways to maintain operational reliability is the organization of effective monitoring of the operating equipment status. Therefore, to maintain the required operational reliability of transformers, it is very important to assess the technical condition, quality maintenance of transformers, organization of effective monitoring of the operating equipment state, diagnostic control and accurate identification of the failures causes. That's why, in recent years, particular attention has been paid to monitoring and diagnostics of the main electrical equipment of power systems [10-17].

During operation of the transformer, the oil in it undergoes profound changes. There are changes in chemical and electrophysical indicators that characterize the working capacity of the oil $[9,12,15,16,22-24]$. Its insulating properties deteriorate, sediment accumulates on the active part, which complicates heat removal, aging of cellulose insulation is accelerated and its insulating properties deteriorate. The degradation of the oil is accelerated at elevated temperatures of the transformer, the presence of contact with the oxygen of the air, a strong electric field, in the presence of various materials from which the transformer is made. The dominant factor in the degradation of transformer oil are oxidative transformations of its constituent hydrocarbons. With an increase in the concentration of oxygen in the oil, the rate of degradation increases [3,18$21,23,25,26]$.

\section{Methods}

For research on changes in transformer oil (TO), photographing of the researched object is carried out simultaneously in transmitted and scattered radiation. Each oil sample was placed in a quartz cuvette (Figure 1) and alternately irradiated with a narrow beam of light 1 , with the result that we could visually simultaneously observe the scattered radiation and the radiation transmitted through the oil on a white screen 5 located at an angle of $45^{\circ}$ to the incident radiation 1 on a cuvette. The light source was a light-emitting diode with a white radiation spectrum.

For research, 4 samples of GK oil with different degrees of degradation and with different acid numbers were taken. Sample No. 1 has $K=0.0073$ - relatively pure TO, sample No. 2 with $\mathrm{K}=0.035$, sample No. 3 $\mathrm{K}=0.057$ - it is TO with an average degree of degradation, sample No. 4 with $\mathrm{K}=0.12$ TO with an extremely high degree of degradation.

Corresponding author: beautiful_nut@mail.ru 


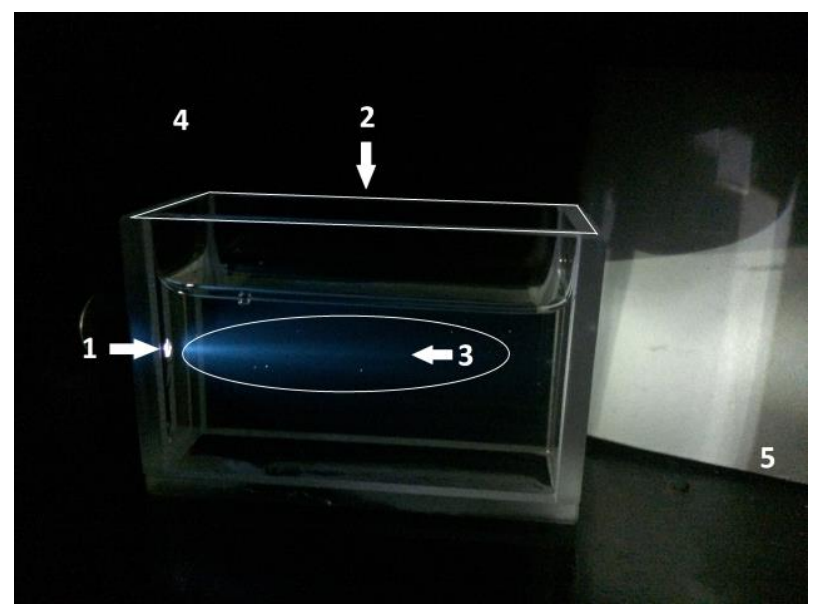

Fig. 1. Experimental setup : 1- source of complete light (white light-emitting diode); 2- $5 \mathrm{sm}$ quartz cuvette; 3- scattered radiation in the oil, that is observed at an angle of $90^{\circ}$ to the incident radiation; 4- black screen to eliminate rereflection of scattered radiation; 5- white screen, fixed at an angle of $45^{\circ}$, for observing the transmitted radiation.

\section{Results}

Figure 2 shows the image of transformer oil in scattered light (Figure 2 a) and the image of transmitted light on a white screen (Figure $2 \mathrm{~b}$ ) transformer oil with an acid number $\mathrm{K}=0.0073$.

Figure $2 \mathrm{~b}$ shows the transmitted radiation having a greenish color. In Figure 2 a there is a weak scattering, the color is purple. The color of the transmitted radiation is complementary to the color of the scattered radiation, which indicates the absence of absorption in the oil and the attenuation of the transmitted radiation is due only to Rayleigh scattering on colloidal particles whose sizes do

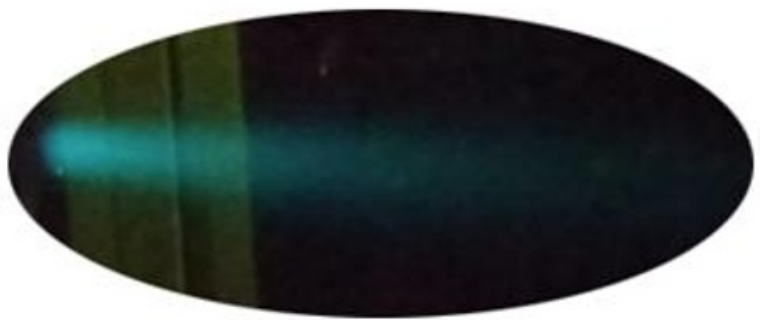

a) not exceed $70-80 \mathrm{~nm}$. All this demonstrates that TO is pure.

Figure 3 shows the transformer oil with the acid number $\mathrm{K}=0.035$. The acid number increased, that is, there were changes in transformer oil that are visible in the transmitted and scattered light. The color of the scattered radiation is blue-violet (Figure 2 a), the color of the transmitted radiation is orange (Figure. $2 \mathrm{~b}$ ), which is not completely additional and indicates the presence of absorption in the ultraviolet area of the spectrum, which is caused by an increase in the number and size of aromatic compounds in oil. Changes in the color of scattered radiation due to an increase in the size and concentration of colloidal particles.

Figure 4 shows the transformer oil with the acid number $\mathrm{K}=0.057$. From this figure $4 \mathrm{a}$ it is shown that the color of the scattered radiation turned blue-green and a green spot appeared at the beginning of the beam. Transmitted radiation became yellowish-brown. (Figure $4 \mathrm{~b}$ ), which already indicates the presence of oxidized colloidal particles of a larger size than in the previous two samples (Figure 3 and Figure 4). The presence of a green spot at the beginning of the scattered light beam indicates the appearance of three to four ringed aromatic compounds in the oil, which luminesce well due to a significantly larger molecular absorption cross section than the Rayleigh scattering particle section, which gives off a luminescence spot on the background of the scattering beam.

Figure 5 shows the transformer oil with the acid number $\mathrm{K}=0.12$. It is seen that the transmitted radiation already has a dark brown color (Figure $5 \mathrm{~b}$ ), and this also indicates an increased number of oxidized particles and an increase in their diameter. The luminescence at the input is observed very powerful (Figure $5 \mathrm{~b}$ ), its color is yellow, which indicates a quantitatively and qualitatively

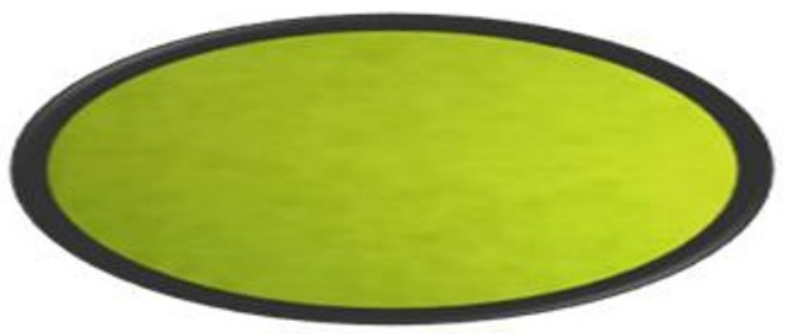

b)

Fig. 2. Transformer oil with the acid number $K=0.0073$ : (a) - scattered radiation; (b) - transmitted radiation.

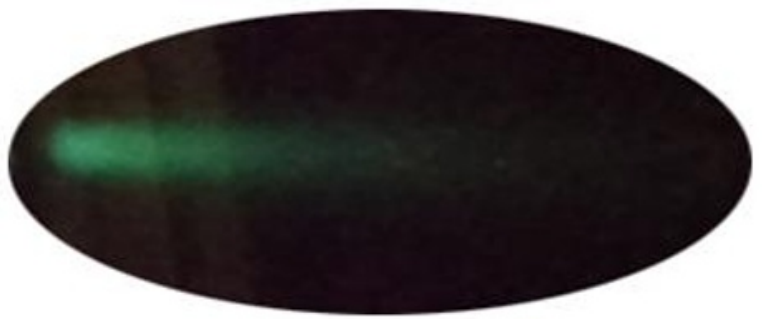

a)

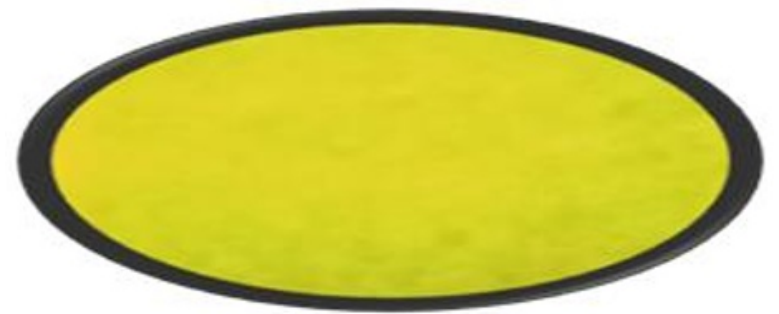

b)

Fig. 3. Transformer oil with the acid number $K=0.035$ : (a) - scattered radiation; (b) - transmitted radiation. 


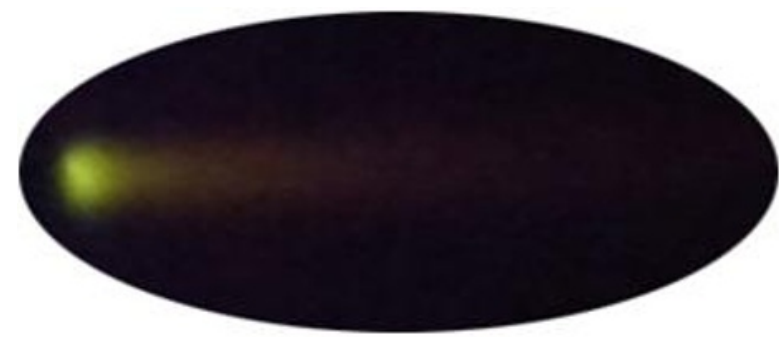

a)

Fig. 4. Transformer oil with the acid number $\mathrm{K}=0.057$.

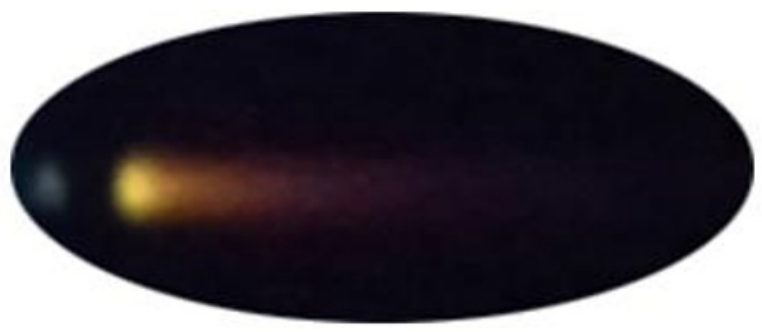

a)

Fig. 5. Transformer oil with the acid number $\mathrm{K}=0.12$.

increased aromatic compounds.

The basis of the insulating oil is the naphtheneparaffin fraction. These compounds have weak absorption bands in the visible range, as a result of which the fresh oils look like transparent and almost colorless liquids. Taking into account the weak absorption coefficient, the thickness of the oil layer (cuvette thickness) must be at least $10 \mathrm{~mm}$. In most researches of the transmission spectrum (absorption) of oils should be a cuvette with a thickness of $50 \mathrm{~mm}$. To clarify the characteristics of the chemical composition of fresh oils, transmission spectra are obtained using a 25 mm cuvette.

In the process of aging (oxidation) of oils, degradation products accumulate in them. Radiation absorption of pure (fresh) oils in the range of 300-600 $\mathrm{nm}$ is primarily due to resinous-asphaltenic substances present in the oil, whose influence begins to noticeably affect at wavelengths $\lambda$ less than $500 \mathrm{~nm}$. Vaseline oil contains neither aromatic compounds (only at the level of traces), nor, especially, asphaltenic substances. In this regard, in the range of $300-500 \mathrm{~nm}$, this oil does not absorb radiation. In the range of $600-800 \mathrm{~nm}$, there is no appreciable absorption from all components of the original insulating oils.

The total transmission of oils with increasing oxidation decreases. On the lumen the oil becomes darker, turbidity appears. The oxidation process is accompanied not only by changes in the chemical composition of oils, but also by the formation of a dispersed phase, since polar molecules of oxidation products, for example, naphthenic acids, tend to associate. The consequence of these processes is the scattering of radiation transmitted on the oil. For the considered spectral range, the particle sizes of the dispersed phase are more than an order of magnitude

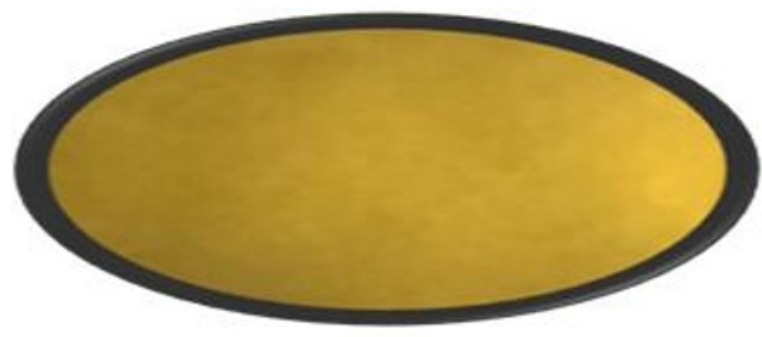

b)

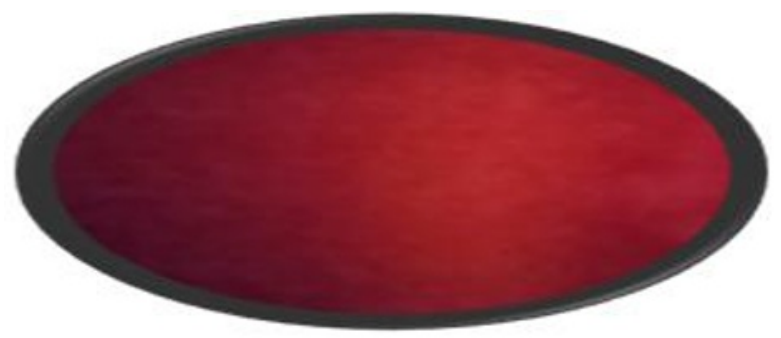

b)

smaller than the wavelength and the scattering intensity on these particles is described by the Rayleigh law: (I $\sim 1$ $\left(\lambda^{4}\right)$. As a result, in the short-wave part of the spectrum, the absorption of radiation is the strongest.

Further oxidation of oils leads to an increase in the size of colloidal particles, which leads to some influence of the effect of scattering on the long-wave part of the transmission spectrum - $\lambda^{4} \sim 800 \mathrm{~nm}$. However, a significant increase in the size of colloidal particles in oil is limited by the process of their precipitation.

In the deep oxidation of oils, another factor affecting the decrease in the transmittance of oils is the accumulation of resinous-asphaltenic substances with a recondensed aromatic structure in insulating oils. The boundary of intense absorption of these compounds is shifted to the middle part of the visible range. Further oxidation of the oils leads to an increase in colloidal particles.

Oil aging under discharge-arc processes is different from thermal processes. During discharge processes in oil, first of all, burning products with sizes of about 500 $1000 \mathrm{~nm}$ and more are formed. With such particle sizes in the spectral range of 300-800 nm, practically uniform scattering of radiation occurs. In contrast to the thermooxidative processes, the "collapse" of the spectral characteristics in the short-wave part of the spectrum does not occur.

\section{Discussion}

It is possible to determine the size of the oxidized particles in the transformer oil by determining the color of the scattering and the depth of its infiltration in the oil in a visual way. And it is possible to determine number of particles, as well as the acid number of the transformer oil by determining the intensity of the 
scattering. According to the brightness of the luminescence at the radiation input in the oil, it is possible to judge the concentration of aromatic compounds in transformer oil and the qualitative change in the luminescent molecules.

Consequently, this diagnostic system allows assessing the condition of power transformers without shutting them down, making decisions on their further operation, detecting thermal and discharge defects, that will make it possible to predict the possibility of further trouble-free operation of power transformers for a certain period of time, which is very important and relevant nowadays.

\section{References}

1. N.F. Kotelets, N.A. Akimova, M.V. Antonov, Testing, operation and repair of electrical machines, 383

2. T.T. Baltynov, Research of failures of power transformers during operation, Modern equipment and technologies: Collection of works of the XVII Int. Scientific and Practical Conf. of Students, Postgraduates and Young Scientists, 18-22 (2011)

3. O.A. Turanova, A.E. Vendyukov, L.G. Gafiyatullin, V.K. Kozlov, Research of the mechanism of sediment formation in transformer oil, Chemistry and technology of fuels and oils, 4, 628-631 (2013)

4. M.Y.Lvov, Analysis of damage to power transformers of $110 \mathrm{kV}$ and above, JSC IDGC Holding. Travec Conf. (2009)

5. G.A. Muratayeva, I.A. Muratayev, Application of the temperature dependence of the dielectric loss tangent to assess the aging of transformer oil, Actualscience, 1-3(3), 70-72 (2015)

6. M.V. Dagayeva, D.V. Kataseva, A.S. Katasev, A.P. Kirpichnikov, Fuzzy expert diagnostics system for oil-filled power transformers. Bulletin of the Technological University, 21(2), 148-154 (2018)

$7 . \quad$ V.V. Petrova, V.F. Novikov, R.R. Garifullina, Problems of transformer oil sample preparation. In the digest: fundamental and applied research: problems and results, Materials of the Int. scientific-practical conference dedicated to the 100th anniversary of the FSBEI of HE "GSSTU named after academician Millionshchikov M D, 2,118-120 (2018)

8. Ya.M. Kashin, G.A. Kirillov, A.B. Varenov, A.A. Yermolaev, V.E. Gabidulin, Analysis of modern methods and equipment for quality control of transformer oil, Bulletin of the Adyghe State University. Series 4: Natural Mathematical and Technical Sciences, 3(206), 109-121 (2017)

9. S.P. Vysogorets, Development of methods for rapid analysis of the quality parameter of insulating oil, Reliability and safety of energy, 11(1), 41-47 (2018)

10. R.R Abdrashitov, Diagnostics and monitoring of power transformers. In the digest: The role and place of information technology in modern science, Collection of articles of the Int. Scientific and Practical Conf., 19-21 (2018)

11. T.P. Vagapov, T.A. Volkova, Transformer oil as a diagnostic medium for power transformers, In the digest: Electrotechnical complexes and systems materials of the int. scientific-practical conf. (2016)

12. A.I. Syrtsov, D.V. Polovinka, O.S. Parsentev, Increasing the reliability of power transformers by improving their tests, Prospects for the development of information technology, 32, 76-86 (2016)

13. V.P. Tomin, Ye.N. Korchevin, Research of the transformer oils aging in the atmosphere of various gases under the influence of an electric field, Energetic, 5, 25-28 (2017)

14. A.A. Kartashov, V.F. Novikov, Determination of furan compounds in transformer oil by gas chromatography using new sorbents. Proceedings of higher educational institutions, Energy problems, 1-2, 46-52 (2016)

15. V.V. Petrova, Results of long-term periodic diagnostics of power transformers, In the digest: Int. Collection of scientific articles XLVII Int. correspondence scientific and practical conference, 34-37 (2018)

16. A.Ye. Obukhov, L.L. Chaikov, K.V. Kovalenko, S.V. Krivokhizha, A.N. Lobanov, Dynamic light scattering method and complex spectral atomic-molecular identification of the structure of colloidal formations in transformer oil, Actual problems of oil and gas, 2(14), 22 (2016)

$17 . \quad$ L.G. Gafiyatullin, Yu.K. Bikkinyaeva, A.N. Turanov, V.K. Kozlov, Features of the degradation of olive oil according to UV-spectroscopy, the magazine Energy of Tatarstan, 9-10, 138-140 (2013)

18. Yu.K. Bikkinyaeva, A.N. Turanov, V.K. Kozlov, O.A. Turanova, Prospects for the development of chromatographic analysis of gases dissolved in oil and other analytical methods used to diagnose the state of oil-filled transformers, University news. Problems of Energy, 9-10, 148$152(2014)$

19. Yu.K. Ilyasova, Yu.K. Bikkinyaeva, A.N. Turanov, V.K. Kozlov, O.A. Turanova, Analysis of optical spectra of transformer oils in the area of 850-1000 nm, University news. Journal Problems of Energy, 7-8, 13-17 (2018)

20. V.K. Kozlov, M.Sh. Garifullin, Monitoring the status of transformer oils according to their optical spectra, New in the Russian electric power industry, 1, 16-22 (2017)

21. O. Shutenko, O. Proskurnina, V. Abramov, Comparative analysis of risks which are accompanied by the use of typical and boundary gases concentrations for the diagnostics of high voltage transformers, Energetika, 64(3-29), 137-145 (2018)

22. G. Poiss, S. Vitolina, J.M. Riga, Advances in Science, Technology and Engineering Systems Journal, 3(1),148-154 (2018) 
23. R. Paul, E.M. Ohodnicki, Chemical Sensing Strategies for Real-Time Monitoring of Transformer Oil, IEEE Sensors Journal, 17(18), 5786-5806 (2017)

24. J. Singh, Y.R. Sood, P. Verma, Impact of Accelerated Stresses on Power Transformer Insulation, Energy and Power Engineering, 09(04), 217-231 (2017)

25. L. Liu, H. Wu, T. Liu, H. Feng, H. Tian, Z. Peng, Influence of moisture and temperature on the frequency domain spectroscopy characteristics of transformer oil, Proceedings of the IEEE International Conference on Dielectrics, 1, 565-568 (2016)

26. O.E. Kurakina, V.K. Kozlov, O.A. Turanova, A.N. Turanov, Research of the Changes in the Structural Group Composition of Transformer Oil During Operation, Problemele energeticii regionale, 2, 37 (2018) 